\title{
An adaptive behavioral immune system: a model of population health behavior
}

Julian M. Saad (10 ${ }^{1 凶} \&$ James O. Prochaska ${ }^{1}$

The understanding that immunity could be strengthened in the general population (e.g., through vaccine interventions) supported global advances upon acute infectious disease epidemics in the eighteenth, nineteenth, and twentieth centuries. However, in the twenty-first century, global populations face chronic disease epidemics. Research demonstrates that diseases largely emerge from health risk behavior. The understanding of how health behavior, like the biological immune system, can be strengthened in the general population, could support advances in the twenty-first century. To consider how health behavior can be strengthened in the general population, the authors present a theoretical model of population health behavior. The model operationalizes health behavior as a system of functions that, like the biological immune system, exists in each member of the population. Constructs are presented that operationalize the specific decisions and habits that drive health behavior and behavior change in the general population. The constructs allow the authors to present parallels (1) among existing behavior change theories and (2) between the proposed system and the biological immune system. Through these parallels, the authors introduce a model and a logic of population-level health behavior change. The Adaptive Behavioral Immune System is an integrative model of population health behavior.

\footnotetext{
${ }^{1}$ Cancer Prevention Research Center, The University of Rhode Island, Kingston, RI, USA. ${ }^{凶}$ email: jsaad@uri.edu
} 
C onsistent references to a human "immune system" did not emerge until researchers in the second half of the twentieth century proposed that integrated functions (rather than separated biochemical/immunological responses) facilitate immunity (Moulin, 1989). Steinberg et al. (1981) published the first collected works on the human immune system with a proposed emphasis on a system of integrating functions. Around this time, the field of psychoneuroimmunology conducted research on the integration of biological, behavioral and psychological functions. Solomon and Moos proposed an initial integration of psychological functions, stress, and autoimmune markers (Solomon and Moos, 1964; Solomon and Moos, 1965; Solomon, 1969; Solomon, 1981); and Ader and Cohen, 1974; Ader (1980) demonstrated that immune responses (i.e., antibody production) and behavioral responses (e.g., taste aversion) could be classically conditioned within the same exposure. Collectively, this research demonstrated that biological and behavioral functions maintain integrative relationships that govern health-and-wellbeing. The works of the late twentieth century also demonstrated that advances to theory and practice can occur when functional and integrative approaches to health research are taken.

In the twenty-first century, there remains a continued need to understand relationships among biology, behavior and population health. Recently, Schaller and Duncan (2007) proposed a Behavioral Immune System (BIS) to explain relationships between acute, infectious disease-preventing behaviors, defense behaviors, and stress in the population. The model proposes psychological mechanisms that explain relationships among reflexive behaviors (e.g., disgust-responses) and the detected costs of immunological defenses (Schaller and Duncan, 2016). The authors propose that the BIS consists of functions that (1) detect cues indicating the presence of acute infectious pathogens in the environment, (2) initiate disease-relevant affective and cognitive functions, and (3) activate behaviors that avoid (the perceived threat of) pathogenic infection (Schaller and Park, 2011). It has been suggested that the functions of BIS are evidence of psychoneuroimmunology at work in the population (Clark and Fessler, 2014). The model indicates how behavioral functions and biological functions integrate to support the prevention of acute infectious diseases (Schaller nd Duncan, 2016).

However, another class of diseases also threaten population health. Despite recent outbreaks of the infectious COVID-19, chronic diseases remain the leading cause of mortality (Chartier and Cawthorpe, 2016) and they have strong relationships to human behavior. Epidemiological research has identified that a small number of behavioral risk factors (e.g. smoking, alcohol misuse, unhealthy diet, inactivity), rather than environmental risk factors (e.g., exposure to infectious pathogens) are the leading cause of disability-adjusted life years (DALYs) and premature deaths in populations (Mokdad et al., 2018). Health behavior plays an important role in the prevention of both acute and chronic disease (Kretzer and Larson, 1998). This crisis beckons the need for a model that can account for relationships between disease and behavior. The present manuscript proposes a behavioral immune system, an Adaptive Behavioral Immune System (ABIS), as a model of population health behavior. Similar to the collected works presented by Steinberg et al. (1981) the emphasis within this proposal is on integrated functions rather than separated structures, constructs, or behaviors.

\section{Part 1: a need for a systemic model of health behavior}

Recent reports suggest that only a small number of behaviors are accounting for the large majority of chronic disease, disability, and premature death (Mokdad et al., 2018; Berrigan et al., 2003; Katz et al., 2005; Katz, 2009; Katz et al., 2017; Li et al., 2018;
Dreyzehner, 2017). Accounting for the current death toll in the US alone (as cited in Mokdad et al., 2018; CDC, 2018), Dietary Risks ( 529,000 deaths), Smoking ( 490,000 deaths), Low Physical Activity ( $\sim 90,000$ deaths), and Alcohol Misuse $(\sim 88,000$ deaths) stand together as a big problem-the "Big 4." At the biological level, these risk behaviors can also put individuals and populations at risk for contracting and/or spreading acute infectious diseases (Kretzer and Larson, 1998). At the social level, one can see advertising for cigarettes, alcohol, and fast food in underserved, urban communities. Accessibility to these behaviors supports the rising rates of chronic disease in the underserved (D'Angelo et al., 2016). Although the Big 4 have received attention in the past decades (Dreyzehner, 2017; Mokdad, 2004), a concerted effort to bring them to the forefront of population health innovation and intervention is still needed.

Conceptualizing the big problem: integrating the "Big 4". While a proposed integration of biochemical/immunological functions supported advances in the twentieth century, a proposed integration of the behavioral functions that drive the Big 4 might support advances in the twenty-first century. There is no avoiding the systemic nature of the Big 4 in population health. High-risk, high-cost populations engage in multiple unhealthy behaviors (Li et al., 2018; Prochaska and Prochaska, 2011; Lippke et al., 2012), and research has demonstrated that a large majority of adults have at least two of the Big 4 (Berrigan et al., 2003). There remains an increasing need for theory-driven methods of systematizing and conceptualizing patterns in co-occurring behaviors (Lippke et al., 2012).

Until now, the behaviors of the Big 4 have largely been studied, analyzed and treated separately. Separate disciplines, grant funding structures, and research groups have assembled to investigate the implications of separate health behaviors upon population health outcomes. However, in our Philosophy of Health (Saad \& Prochaska, 2020), we propose that health behavior can also be observed as a system of decisions-andhabits. Decisions are seen as functional when they are precise and can be used to prioritize and organize changes for a behavior. Habits are functional when they evidence a functional ease or variability in ones patterns of breathing, drinking, eating and moving. In A Philosophy of Health (2020), decisions are responsible for precision in health behavior; and habits are responsible for variation in healthy behavior. This paper will build upon A Philosophy of Health (2020) to present a model of population health behavior: an Adaptive Behavioral Immune System.

\section{Part 2: behavior change as "behavioral immunity"}

This paper advances upon our Philosophy of Health's (2020) parallels of behavior change and biological immunity, suggesting that healthy, maintained behavior change can be best understood as a strengthening of behavioral immunity. To do this, we present how behavior change and biological immunity exhibit common pathways to change through the functions of variation and precision (Saad and Prochaska, 2020):

1. Variation is observed in a system's range of abilities, the "varied-abilities", that maintain health in presently changing conditions.

2. Precision is observed when a system prioritizes and organizes variations that maintain health in future, changing conditions.

In biological immunity, functional variation in the microbiome supports and reinforces the development of functional hostdefense responses. Complementarily, functional precision of hostdefense system responses prioritizes functional variability in the 


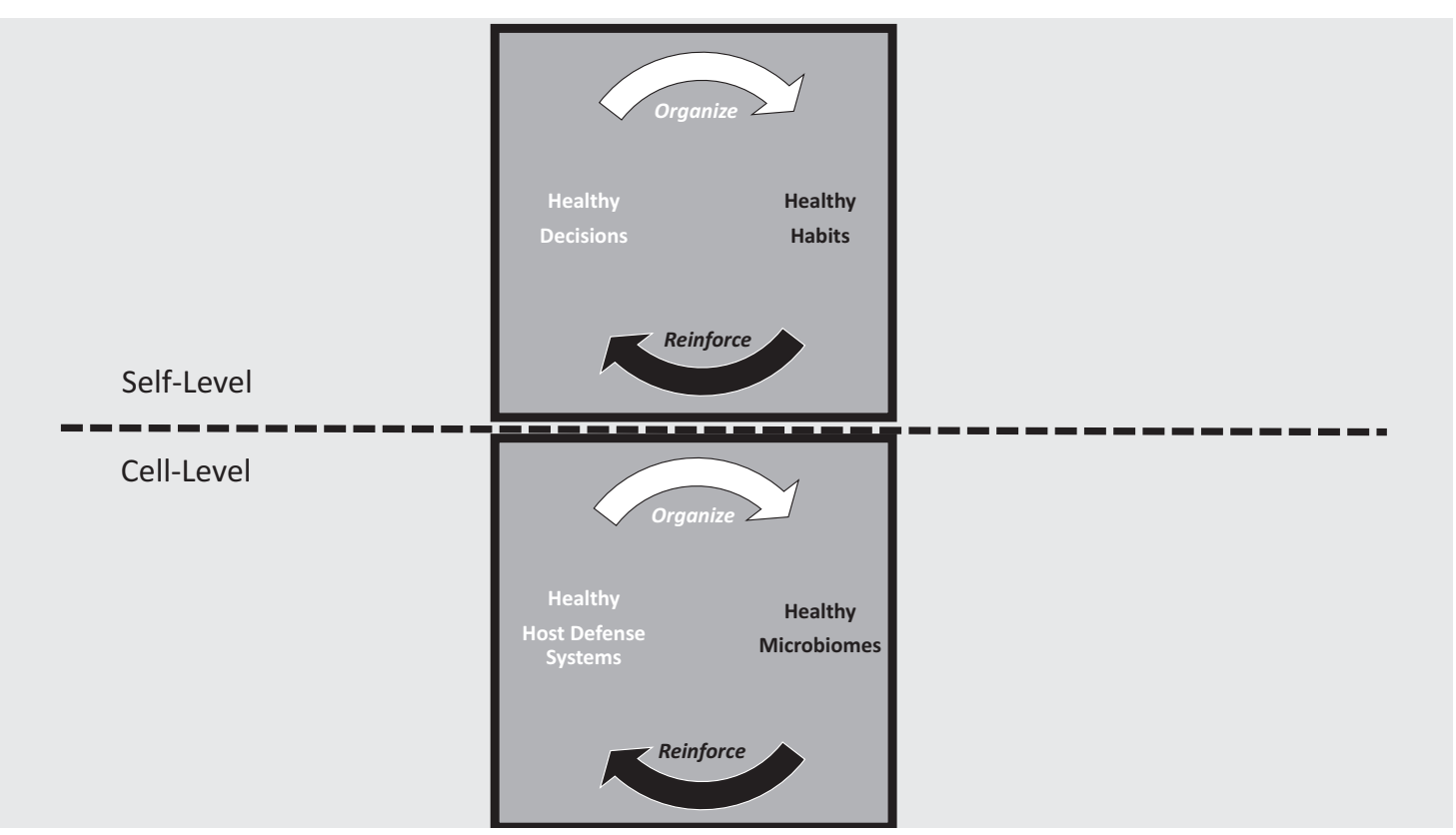

Fig. 1 Reciprocal functions at multiple levels. This model of health demonstrates how the complementary functions integrate for a whole system. Functional precision organizes variation. Functional variation reinforces functional precision. When integrated, the functions can serve health from multiple levels.

microbiome (Saad and Prochaska, 2020). New microorganisms enter the human microbiome from the environment each moment, leading it to vary from moment to moment. After detecting these changes, the host-defense system can act to prioritize functional variation in the microbiome so that the colonies are balanced. If increasing variability in the microbiome is maintained by the host-defense response, without the spread of an infection, this host-defense response is reinforced. Why? Healthy microbiomes, support the health of a physical body; and that body produces new, healthy immune cells. When functional, variation in the microbiome and precision of the host-defense system reciprocally integrate to support biological immunity (see Fig. 1).

In behavior change, functional variation in one's habits (i.e., healthy habitual patterns of breathing, drinking, eating, and moving) supports and reinforces the development of functional decisions. Complementarily, the precision of good decisions prioritizes functional variability in ones habits, leading to the development of healthy "lifestyle". New environmental conditions (e.g., changes in temperature) impact our habits (e.g., our rhythms of breathing) each moment, leading them to vary from moment to moment. When we detect these changes, our decisions can act to prioritize functional variations in our habits so that our "lifestyle" is balanced. If variability in our habits is maintained by our decisions without the spread of an addiction, our decision-making is reinforced as "good". Why? Healthy habits, support healthy lifestyles that reinforce (i.e., make easier) healthy decisions. When functional, variation in habits and precision of decisions reciprocally integrate to support behavioral immunity (see Fig. 1).

In sum, precision and variation reciprocally integrate to produce healthy Cells and healthy Selves. At the level of the Cell, host-defense systems organize the microbiome so that variability is functional (i.e., diverse microbial communities cohabitate without excess conflict/infection spread). Reciprocally, functional variability in the microbiome also reinforces healthy host-defense system responses because rich cohabitating microbiomes support nutrient metabolism and homeostasis for the entire body (Clemente et al., 2012; Rothe and Blaut, 2012; Blaut and Clavel, 2007; De Vadder et al., 2014). At the level of the Self, healthy decisions organize one's system of habits so that habits functionally vary (e.g., one has a variable/diverse lifestyle that is free from addictions but has healthy routines). Reciprocally, this functional variability in habits also reinforces healthy decisionmaking because healthy lifestyles make decision-making easy. The reciprocal integration of precision and variation will help us to see functional states of immunity from multiple levels. Their fragmentation will help us to see common, dysfunctional states of immunity (discussed in the coming sections).

The functions of precision and variation help to establish concrete parallels between the functions of behavioral and biological immunity seen in Table 1. Before going more in-depth into the parallels within models of behavioral and biological immune systems (seen in Table 2), we will first develop how the precision-of-decisions and variation-in-habits support a generalizable model of population health behavior. Now we view health behavior as a system, an immune system, of reciprocally integrating decisions and habits is novel.

Variation in habits (and habitual life functions). Smoking, alcohol abuse, unhealthy diet, and inactivity are not just separate behaviors. They also evidence dysfunctional patterns of four integrating, habitual functions: breathing, drinking, eating, and moving. When healthy, habitual patterns of breathing, drinking, eating, and moving evidence a natural ease of functioning in a human life. Healthy breathing is relaxed and variable; healthy drinking and eating reflect variability in the diet; and healthy moving is fluid and variable. Conversely, the Big 4 evidence maintained-dysfunctional patterns or variations of these functions. Chronic smoking creates and maintains dysfunctional variations of the breathing function. Alcohol abuse creates and maintains dysfunctional variations of the drinking function. Unhealthy diets create and maintain dysfunctional variations of the eating function. Inactivity creates and maintains dysfunctional variations of the moving function. When observing functional rhythms or variations in these fundamental functions one observes an individual's ease-of-functioning in the present moment (Saad and Prochaska, 2020). When observing dysfunctional rhythms or variations in these fundamental functions one may observe a disease in the present moment. 
Table 1 Parallels between models of biological and behavioral immune systems.

\begin{tabular}{|c|c|c|c|c|c|}
\hline Immune system & Level of functioning & Precision function & $\begin{array}{l}\text { Variation } \\
\text { function }\end{array}$ & Functional precision & Functional variation \\
\hline $\begin{array}{l}\text { Biological } \\
\text { immune system }\end{array}$ & The cell & $\begin{array}{l}\text { The host- } \\
\text { defense system }\end{array}$ & $\begin{array}{l}\text { The human } \\
\text { microbiome }\end{array}$ & $\begin{array}{l}\text { The host-defense system } \\
\text { prioritizes symbionts and } \\
\text { organizes the removal of } \\
\text { pathogens and parasites }\end{array}$ & $\begin{array}{l}\text { Microbial variability/ } \\
\text { diversity in the } \\
\text { microbiome }\end{array}$ \\
\hline $\begin{array}{l}\text { Adaptive behavioral } \\
\text { immune system }\end{array}$ & $\begin{array}{l}\text { The "Self" (i.e. the } \\
\text { individual } \\
\text { human being) }\end{array}$ & Decisions & Habits & $\begin{array}{l}\text { Decisions prioritize good habits } \\
\text { and organize the removal of } \\
\text { bad habits }\end{array}$ & $\begin{array}{l}\text { Variability in one's } \\
\text { healthy habits (i.e. } \\
\text { "healthy lifestyle") }\end{array}$ \\
\hline
\end{tabular}

Table 2 Parallels within models of biological and behavioral immune systems.

\begin{tabular}{|c|c|c|c|c|c|}
\hline $\begin{array}{l}\text { General } \\
\text { function }\end{array}$ & General responsibility & $\begin{array}{l}\text { Specific } \\
\text { functions }\end{array}$ & Definition of specific function & $\begin{array}{l}\text { Evidence in the biological } \\
\text { immune system }\end{array}$ & $\begin{array}{l}\text { Evidence in the adaptive } \\
\text { behavioral immune system }\end{array}$ \\
\hline \multirow[t]{3}{*}{ Precision } & $\begin{array}{l}\text { The functions that } \\
\text { prioritize and organize } \\
\text { rhythms or variations }\end{array}$ & Detection & $\begin{array}{l}\text { The cost-benefit analyses that set } \\
\text { a point of reference from which to } \\
\text { reliably change functioning }\end{array}$ & $\begin{array}{l}\text { Detection and pattern recognition } \\
\text { functions performed by toll-like } \\
\text { receptors }\end{array}$ & $\begin{array}{l}\text { The weighting of pros/cons } \\
\text { in TTM's Decisional Balance }\end{array}$ \\
\hline & $\begin{array}{l}\text { that sustain health in } \\
\text { future environments }\end{array}$ & Direction & $\begin{array}{l}\text { The sequencing of events within } \\
\text { the system that support } \\
\text { movement (from a detected point } \\
\text { of reference) towards a future } \\
\text { state of healthy functioning }\end{array}$ & $\begin{array}{l}\text { A coordination of immune events } \\
\text { following the detection of an } \\
\text { antigen; A coordination of } \\
\text { immune events following the } \\
\text { detection of self-antigens in } \\
\text { autoimmune patients; }\end{array}$ & $\begin{array}{l}\text { The sequencing of behavior } \\
\text { change events to create } \\
\text { movement through TTM's } \\
\text { five Stages of Change }\end{array}$ \\
\hline & & Selection & $\begin{array}{l}\text { The conditioning of precise } \\
\text { responses that readily replace } \\
\text { dysfunctional conditions with } \\
\text { functional conditions (to maintain } \\
\text { direction). }\end{array}$ & $\begin{array}{l}\text { The functioning of enzymes and } \\
\text { antibodies to tag, neutralize, and } \\
\text { remove pathogens }\end{array}$ & $\begin{array}{l}\text { The use of TTM's Processes } \\
\text { of Change to tag, neutralize, } \\
\text { and replace bad habits }\end{array}$ \\
\hline Variation & $\begin{array}{l}\text { The functions that } \\
\text { express the "varied- } \\
\text { abilities" that evidence } \\
\text { health in presently } \\
\text { changing environment }\end{array}$ & $\begin{array}{l}\text { (to be } \\
\text { addressed } \\
\text { in future } \\
\text { work) }\end{array}$ & & $\begin{array}{l}\text { The human microbiota: the } \\
\text { systemic colonies of } \\
\text { microorganisms that continuously } \\
\text { vary to sustain life of the } \\
\text { biological tissue }\end{array}$ & $\begin{array}{l}\text { Breathing, drinking, eating, } \\
\text { and moving: the habitual life } \\
\text { functions that continuously } \\
\text { vary to sustain life in the } \\
\text { human body }\end{array}$ \\
\hline
\end{tabular}

The integrated consideration of breathing, drinking, eating, and moving as habitually varying, life functions (rather than Smoking, Alcohol Misuse, Unhealthy Diet, and Inactivity) supports an integration of multiple health behaviors that is generalizable to the population. Not all humans smoke cigarettes; but, all humans must breathe to exist. Not all humans drink excessive amounts of alcohol; but, all humans must drink to hydrate. Not all humans consume unhealthy amounts of processed sugar; but, all humans must eat to nourish. Not all humans sit for prolonged amounts of time; but, all humans must move to maintain vitality. While other forms of habitual functioning exist (e.g., as seen during illicit IV drug addiction; or during non-suicidal self-injury), variations of breathing, drinking, eating and moving are fundamental functions that sustain life for all humans. It should come as no surprise that creating and maintaining dysfunctional variations of these functions will fundamentally compromise life and therefore health in a general population.

The precision of decisions (and executive functions). When the behavior change of a risk behavior begins, a decision is made and a series of decision-making practices must follow. Good decision-making is used so that those patterns of breathing, drinking, eating and moving can be practiced and therefore maintained into the future (Saad \& Prochaska, 2020). Humans have the ability to employ decision-making processes that prioritize and organize functional variations habits (e.g., removing habitual shallow breathing by prioritizing, intentional deep breathing each morning; removing habitual sugar binging by prioritizing fiber-rich carbohydrates during each meal). While habitual variations of breathing, drinking, eating and moving can operate below the level of conscious awareness, conscious decisions or executive functions (e.g., attention and pattern recognition) integrate with habits to prioritize and organize longerterm change.

Precision-of-decisions and variation-in-habits make behavior a system. The first differentiation of decision and habit within a health behavior system was done in our Philosophy of Health (Saad and Prochaska, 2020). Decisions evidence a precision that can prioritize and organize variations in healthy behavior. Habits evidence this variation in health behavior that is in flux from moment to moment, providing real time data on trajectories of health behavior. Together, the precision of decisions and the variation in habit allow health behaviors to integrate as a system.

In the current proposal for an Adaptive Behavioral Immune System (ABIS), we build upon this system of thought. We present specific forms of decisions in health behavior change in parallel with specific forms of host-defense functions during biological immunity. To build upon A Philosophy of Health, we will demonstrate how the precision of decisions and the precision of host-defense functions evidence common patterns changes in population health. These parallels will support the view that healthy behavior change is best understood as a building of behavioral immunity. 


\section{Part 3: paralleling behavior change and biological immunity}

It is well documented that population-level biological interventions (e.g., vaccine interventions) and social interventions (e.g., social distancing, mask wearing in public) support immunity, prevent disease and save lives. Mathematical models support that disease propagation can be intervened on, and disease transmission can be delayed by measures such as vaccination, social distancing, mask-wearing, and sanitization practices (Eikenberry et al., 2020; Xia et al., 2012; Cheng et al., 2020). These measures support population health. But what else can each individual person do to support their health before a (chronic or acute) disease-state, emerges in their own body? Further, what can a person do to protect their health when a (chronic or acute) disease-state, emerges in their population?

When populations engage in healthy behavior, chronic mental and physical disease can be intervened on (Ornish et al., 1998). When populations engage in healthy behavior, herd immunity can be strengthened because each individual's biological immune system is strengthened (Jesus et al., 2021). Regular practices of healthy breathing, drinking, eating and moving support a healthy lifestyle that supports ease-of-functioning and wellbeing (Saad and Prochaska, 2020). It is well documented that health risk behavior is the greatest predictor of chronic disease (Mokdad et al., 2018); and (2) that healthy behavior can be preventative for both chronic and acute infectious disease (Drela et al., 2004). Despite this evidence, there is not an unified agreement of how population-level interventions should intervene on health behavior to prevent, maintain and reverse both chronic and acute diseases. Health behavior change is a vital tool that is not, but can be, systematically leveraged in population healthcare.

The rise in the chronic diseases (e.g., cancer and autoimmune conditions) in recent years, and the resurfacing of new acute diseases (e.g., COVID-19) beckons the need for a systematic understanding of behavioral health in population-level disease. In this paper we propose a model of a behavioral immune system to address these concerns. We will use higher level abstraction that demonstrates how biological immunity and behavioral immunity are both strengthened.

We propose that three specialized forms of the PrecisionDetection, Direction and Selection-support long-term behavioral and biological immunity. Biological immunity is strong when the host-defense system can:

\section{1. detect threatening variations in microorganisms (e.g., parasites,} pathogens),

2. direct a sequence of immunological events that.

3. select for functional variations to remain in the microbiota.

In parallel, we present (below) how behavioral immunity is strong when decisions can:

1. detect threatening variations in habits,

2. direct a sequence of behavior change events that.

3. select for functional variations in habits.

In the proceedings section we show how Detection, Direction, and Selection form an essential collaboration that develops and maintains functional immunity over time. Table 2 may be referred to as a blueprint for the developing paralells in the proceeding sections.

The Detection of costs and benefits initiates immunity. An immune response is initiated by the detection of (potentially) costly conditions that require change (Zakharova, 2009). When costly conditions are detected, the conditions become a point of reference from which to reliably initiate a change in functioning (e.g., "my smoking is compromising my health, therefore I need to change this behavior"). Given that adaptive responses require resources and therefore, are also costly, the detected benefits/costs of a threat serve as a point of reference from which a system can reliably initiate a change to its current state functioning.

In the biological immune system, responses are initiated when the host-defense system detects costly variations in the colonies of the microbiota (e.g., pathogenic invasion, parasitic infection or acute insult/stressor in tissue) (Zakharova, 2009). In the ABIS, we propose that behavior change is initiated when decisions can detect the costs of unhealthy habits and the benefits of a healthy behavior change. Detection is the cost-benefit analyses that set a reliable point of reference from which to change functioning.

Detection in the biological immune system. The human body houses trillions of microorganisms that systematically inhabit the blood and tissue of the human body. They form the human microbiota. Variation in the microbiota reflects the colonies' abilities to efficiently metabolize complex carbohydrates, convert proteins/neural signals, and the modulate diurnal rhythms that maintain biological homeostasis (Clemente et al., 2012; Rothe and Blaut, 2012; Blaut and Clavel 2007; De Vadder et al., 2014). When a pathogen invades, the efficient rhythms of the microbiota are interrupted. Efficient detection of a threatening variation in a colony (e.g., invasion and infection by bacteria or virus in a tissue) can be the difference between the removal and the spread of an infectious disease. These costly conditions are detected by tolllike receptors, so that an immune response can be initiated (Hoebe et al., 2004; Zakharova, 2009; Takeuchi and Akira, 2010).

Not all invaders are dysfunctional for the biological system. Rather, some are beneficial and become functional inhabitants of the microbiota and functional contributors to health of the human body (Dethlefsen et al., 2007). Inhabitants that are "mutualistic" confer benefits to both the human host and the microorganism; while those that are "parasitic" confer benefits to themselves and (potentially lethal) costs to the human host (Pérez-Brocal et al., 2011). Thus, it is important that the hostdefense system is able to detect the (potential) costs and benefits of both invaders and inhabitants. Given that immune responses are costly (Bonneaud et al., 2003; Råberg et al., 2000), the timely detection of changing conditions in the microbiota is central to the initiation of functional immune responses in the short term that maintain immune-microbial homeostasis over time. Timely detection of a pathogen can be the difference between spread lethal infection and initiation of functional healing.

Detection in an adaptive behavioral immune system. Like in the biological immune system, the timely detection of dysfunctional variations of breathing, drinking, eating, and moving can be the difference between years of chronic disease and years of wellbeing. During behavior change, detection is observed when a health risk behavior is recognized as problematic or costly, and the change of that behavior is recognized as beneficial. It is commonplace for humans to give attention to an issue based upon its costs-and-benefits, or pros-and-cons.

In the ABIS model, the detection of cost-benefits is not a purely rational pattern of thinking. Rather it is reflected in the utility of costs-and-benefits (Thaler, 2018) or the use of Pros-and-Cons (Prochaska and Prochaska, 2016) within the change process. Thaler's (2018) behavioral economics work demonstrates that not all "costs" are perceived as losses. Prochaska et al. (1994) demonstrate that some costs are not "costly enough" when the costs outweigh the pros of change. However, the common saying "I had to hit rock bottom to finally change," is a common way of noting how bad things might need to get to be able to detect the costs of a health risk behavior; and the benefits of its change. The growing research on incentives/rewards in healthcare 
(e.g., Mantzari et al., 2015, Prochaska et al., 2020) is evidence of how increasing the detected benefits of change can help to initiate change from a costly behavior.

Cost-benefit analyses have historically informed behavior change interventions in various population health, medical, and psychotherapeutic settings. Janis (1959) proposed that decisionmaking during conflict could be assessed on a "balance sheet" of risks and benefits prior to the decision. Janis and Mann (1976) operationalized benefits/costs based upon (a) utilitarian gains or losses for self, (b) utilitarian gains or losses for significant others, (c) approval or disapproval from significant others, and (d) selfapproval or self-disapproval. Velicer et al. (1985) created their own Decisional Balance sheet by using principal-components analysis to identify the Pros and Cons of behavior change. The Health Belief Model assesses costs/benefits based upon (1) perceived susceptibility (i.e., the chances of experiencing a risk or getting a condition or disease), (2) perceived severity (i.e., the seriousness of a condition and its consequences), (3) perceived benefits (i.e., efficacy of the advised action to reduce risk or seriousness of impact), and (4) perceived barriers (i.e., belief about the tangible and psychological costs of the advised action) (Rosenstock, 1974). In Cognitive Therapy for Anxiety, costbenefit analyses teach clients to examine the immediate and longterm advantages and disadvantages of assuming exaggerated versus realistic perspectives of anxiety (Clark and Beck, 2011). In Dialectical Behavioral Therapy, Linehan (2014) uses pros and cons to support skills acquisition, behavior change and long-term goal setting. In various models of behavior change, the individual's ability to detect costs/benefits is instrumental for initiating and validating a behavior change.

Literature supports cost-benefit analysis as instrumental for detecting a problem and initiating a healthy behavior change. If a reliable point of reference cannot be set, how can the individual begin to change their behavior? Detection of costs and benefits during population scale interventions has proven to be valuable when conceptualizing the long-term change process across multiple behaviors in varying populations (Prochaska, 1994; Hall and Rossi, 2008). Assessing an individual's ability to detect costs/ benefits not only provides feedback to researchers and clinicians, but is a valuable teaching-point for psychoeducation and behavior change education. In the ABIS model, the detection of dysfunctional habits is central to the initiation of a healthy behavior change.

Sequencing events that create a direction of immunity. In the previous section, we proposed that the detection of costly conditions sets a point of reference from which to change functioning. In this section, we discuss the path of events that are coordinated, or rather, directed following the detection of conditions. Immune systems adapt to continually changing environmental conditions to maintain survival (Zakharova, 2009). However, survival is not a guarantee of health; and not all immune responses maintain an ease-of-functioning over time. A functional sequence of immune events creates a general direction from the point of reference (e.g., detected infection, detected addiction) towards a maintainable-ease of functioning (e.g., immune-microbial homeostasis, meeting national standards for exercise). The maintainable-ease of functioning at the level of biological cells is dependent on the immune system's ability to consistently coordinate pathways that maintain immune-microbial homeostasis (Saad andand Prochaska, 2020; Ruíz-Argüelles, 1996; Kent et al., 1992; Dantzer, 2009). Likewise, maintainable-ease of functioning for one "self" is driven by decisions that sequence a path of behavior change events that maintain a sense of wellbeing and healthy lifestyle.
Direction is the coordination of events that create a path from the detected point of reference towards a future state of functioning.

Direction in the biological immune system. A functional hostdefense system initiates a chain of events that establish pathways of change in the short term that maintain immune-microbial homeostasis over the long-term. One novel area of research is intervening on the sequencing of events, the directions, of an immune system. This is the novel research on messenger RNA (mRNA) vaccine technology. The vaccines work by injecting mRNA, molecule that provides directions to the cells on what types of proteins and responses to build. Through this signaling, important directions on immune responses are communicated (CDC, 2018).

Some experience of short term sickness (e.g., fever, achiness, chills) following exposure to a vaccine. This response itself is evidence of this sequence of internal events in action (RuízArgüelles, 1996; Kent et al., 1992; Dantzer, 2009). The human experience of "sickness" emerges as resources are conserved and re-directed towards the pathway of events that form carry out an immune response (Kent et al., 1992; Dantzer, 2009).

While the immune system sequences immunoregulatory events to costly antigens, (Ruíz-Argüelles, 1996; Medzhitov and Janeway, 2002; Janeway, 1992; Janeway and Medzhitov, 2002), not all antigens are threats. In a dysfunctional immune system, the hostdefense system may detect "self-antigens" (i.e., signals of the body) and direct events that attack the tissue of the human body (Janeway et al., 2014; Naor and Tarcic, 1982). As this dysfunctional path becomes developed (forming an immune pathway), the immune system habitually responds by maintaining chronic inflammation, diminishes ease-of-functioning. A different state of functioning is maintained-autoimmunity. Autoimmune conditions are tangible evidence that the biological immune system can also direct a dysfunctional sequences of events, activating molecules and responses that repeatedly attacking cells of the human body.

When the direction of events coordinated by the host-defense system are functional, inflammation is a functional signal for the sequencing of events that remove a problematic state (e.g., removal of an infection). When the direction of events coordinated by the host-defense system are dysfunctional, inflammation is prolonged (e.g., autoimmunity). Overall, considering the path of immune events from a detected point of reference, towards a future state of functioning (i.e., immunemicrobial homeostasis versus chronic inflammation) provides valuable information on whether the direction of the immune system supports health.

Direction in an adaptive behavioral immune system. Functional decision-making can initiate a chain of events that establish paths of behavior change in the short term that lead to sense of personal freedom (i.e., freedom from addictions/desires) over the longterm. Researchers likely have the best of intentions when conducting behavior change interventions. Yet, some interventions can set a functional point of reference (i.e., "smoking is harming my health-and-wellbeing, therefore I must change this behavior"), but sequence events that move participants away from a desired state of functioning. For example, Hall et al. (1992) intervention events sequenced in a way that lead to increased risk of smoking relapse. Thus, it is not only important to detect a point of reference from which to change behavior; but it is essential that functional sequences of behavior change events sustain movement in the direction of maintainable, not temporary, ease of functioning.

In the behavior change research, various models of behavior change have used continuum, stage, and phase modeling to 
sequence decisions during intervention events that maintain long-term change. Continuum models (as seen in the Theory of Reasoned Action, the Theory of Planned Behavior, and Protection Motivation Theory) use prediction equations and variables to explain intention and likelihood-of-action during behavior change (Armitage and Conner, 2000; Conner and Sparks, 2005; Sutton, 1994, 1998; Schwarzer, 2008). Stage Modeling (as seen in the Transtheoretical Model's five Stages of Change) is used to sequence events, variables and processes based upon defined Stages of Change. Schwarzer (2008) cites that behavior change can be considered in two overarching phases (or "implicit stages"): (1) pre-intentional motivation to support intentions for healthy behavior, and (2) post-intentional volition to support healthy behavior implementation. Phase modeling is exhibited in the Health Action Process Approach (HAPA), which assesses patterns of social-cognitive predictors that support the contemplation process (see Schwarzer, 2008 for an overview of the model). In sum, behavior change models act to coordinate a path of events that (when successfully completed by participants) support movement in the direction of maintainable-ease of functioning.

The selection of conditions that maintains immunity. Changes to functioning can be challenging. Immunoregulation of an infection may lead to the uncomfortable symptoms of inflammation and sickness (Kent et al., 1992; Dantzer, 2009). Behavior change from a bad habit (e.g., smoking addiction) may lead to unpleasant symptoms of distraction, anxiety, and withdrawal. Given that the "side effects" following new changes to functionating can be challenging/costly in the immediate future, it is essential that each system is conditioned to (more) consistently and (more) precisely select for the functional conditions that replace dysfunctional conditions. The consistent Selection of functional conditions that adequately replace dysfunctional conditions helps to maintain movement from a detected point of reference in the direction of health-and-wellbeing. Selection in each immune system is operationalized as the conditioning of responses that replace dysfunction with function to maintain movement toward a future state of functioning.

Selection in the biological immune system. Antibody and enzyme molecules evidence the development of the selection function within the biological immune system. Their release allows the immune system to readily replace dysfunctional conditions (i.e., pathogenic invader in the tissue) with functional conditions (i.e., removal/immunoregulation). When sickness occurs, the immune system produces these molecules/signals to alter cellular activity and activate/upregulate receptors that maintain functional change over the long-term (Ruíz-Argüelles, 1996). Enzymes are nonspecific soluble proteins that create functional conditions by marking and neutralizing pathogens through precise methods: (1) marking pathogens to make them more attractive for hostdefense cells; (2) attracting host-defense cells from the blood; (3) dissolving cell walls of bacteria, so that they lose fluid/minerals and die; (4) destroying parts of a virus directly; or (5) destroying cells infected by viruses (Janeway et al., 2014). Antibodies are specific soluble proteins that bind to specific antigens to tag or neutralize them (Janeway et al., 2014).

Vaccines expose the immune system to a titrated dose of a virus to support selection over time. Following the exposure to a specific virus, the immune system releases antibodies to condition the system's precise responses to that specific virus. This conditioning supports the system's ability to neutralize and/or remove viral conditions when exposed to it again in the future
(Janeway et al., 2014). These responses allow the immune system to work efficiently without systemic infection emerging.

Selection in an adaptive behavioral immune system. Human decision-making is impacted by fluctuations in environmental, social, technological, personal and physiological conditions. The presence of various forms of marketing and media can condition impulsive, rather than healthy decisions. When these responses are conditioned, temporary-ease of functioning replaces maintainable-ease of functioning. To maintain functional decision-making during and after a behavior change intervention (especially during stressful situations such as a viral pandemic), it is necessary that decisions are conditioned to consistently select for healthy behaviors that replace risk behaviors. Healthy behaviors support maintainable-ease of functioning.

Various behavioral science and behavior change science researchers have discussed the importance the proper conditioning of decision-making for of long-term change. Joseph Wolpe (1961, 1968) demonstrated that functional change could be produced through a process of Counterconditioning. Counterconditioning produces change by gradually exposing an individual to anxiety-provoking conditions. This practice is commonly used to change cognitive-behavioral dysfunctions (e.g., phobias and social anxiety) where decision-making/executive functioning falter or is inhibited due to a triggering event. The practice trains the individual to replace dysfunctional responses to the triggering conditions with their own functional responses. Similar to the concept of vaccination, Wolpe's research demonstrated that successful conditioning can result after well-dosed exposure to a stimulus. This method has been used in experimental, clinical, and intervention contexts (Davison, 1968; Prochaska and Prochaska, 2016).

In addition to counterconditioning, other forms of conditioning through reinforcement have been used to condition responses that impact long-term behavior change. Skinner (1953) suggests that therapy introduces reinforcers that compensate for, or correct a history, which has produced a dysfunctional behavior. Bandura (1965) suggests that social reinforcements impact the likelihood of continuing or discontinuing a behavior. Prochaska et al. (1988) found that reinforcement management is contingent on the use of both counterconditioning and stimulus control methods to structure rewards. The Health Belief Model assesses perceived benefits and uses them as reinforcers for healthy change (Janz and Becker, 1984). Janz and Beck (1984) emphasize that reinforcing client's healthy beliefs is a major driver of long-term healthy functioning.

The use of various forms conditioning in behavior change science have provided valuable insight into how ease-offunctioning is maintained throughout time (often despite fluctuations in environmental, social, technological, personal, and physiological conditions). Effective decision-making establishes well-conditioned, stimulus-controlled habits. A major role of behavior change interventions is to guide decision-making to select for functional habits that consistently replace those previously dysfunctional habits (Prochaska and Prochaska, 2016). While enzymes and antibodies are evidence that the biological immune system can mark, neutralize, and replace (potentially) dysfunctional biological conditions; the above behavior change research suggests that decision-making can mark, neutralize, and replace (potentially) dysfunctional behavioral conditions. When an individual can actively replace dysfunctional habits (e.g., through counterconditioning) and reinforce functional habits (e.g., through operant conditioning); it appears that one's decision-making is well-conditioned to select for long-term health behavior change. 


\section{Part 4: observing ABIS operate in one behavior change theory}

By utilizing the constructs Detection, Direction and Selection, we use a higher level of abstraction that goes beyond theories/constructs of behavior and biology to operationalize a common language of immunity. These functions can be uniformly considered in biological immunity because it is already documented that toll-like receptors (Detection) enzymes and antibodies (Selection) work within a coordinated chain of events (Direction) in the biological system to maintain health. However, their unified consideration in behavior change is novel. In this section, we present a unified consideration of Detection, Direction, and Selection by presenting one behavior change intervention model that assesses all three constructs simultaneously. The proceeding sections consider a single behavior change model that (1) accounts for Detection, Direction, and Selection within each of its behavior change interventions; (2) integrates assessments of Detection, Direction, and Selection to predict successful change; and (3) consistently demonstrates interrelationships among Detection, Direction, and Selection in population-level research.

To consider all three constructs the reader can turn to the Transtheoretical Model (TTM). Prochaska and DiClemente's (1983) TTM stands out as a model of change that utilizes the interrelationships among its constructs to guide individual-, group-, and population-level interventions. The TTM constructs (1) Decisional Balance, (2) Stage of Change, and (3) Processes of Change assess (1) Detection, (2) Direction, and (3) Selection respectively during behavior change. These constructs not only satisfy the definitions of each construct, but decades of research have demonstrated their interrelationships as reliable predictors of change.

Detection and direction in existing behavior change research: decisional balance and stages of change in the TTM. Decisional Balance suffices as an assessment of Detection by tracking individuals' weighting of Pros and Cons of Change. The Stages of Change suffice as an assessment of Direction by tracking movement towards healthy functioning (e.g., meeting national standards for weekly exercise; maintained smoking cessation) along the path of five stages that coordinate intervention events. The five Stages of Change are Precontemplation (i.e., no intention to change behavior in the next 6 months); Contemplation (i.e., seriously thinking about changing within 6 months); Preparation (intending to take action in the next month); Action (i.e., actively changing a dysfunctional behavior within the past 6 months); and Maintenance (change continues past 6 months).

There are well documented interrelationships among Decisional Balance and Stages of Change in individual (Prochaska et al., 1994, Prochaska, 1994) and meta-analytic (Hall and Rossi, 2008) research. The movement from Precontemplation (i.e., no intention to change) to Action (i.e., beginning cessation on a quit date), is characterized by a $\sim 1$ SD increase in the Pros, and a $\sim .5 \mathrm{SD}$ decrease in the Cons. These statistical patterns were first found across 12 health risk behaviors (Prochaska et al., 1994) and were later found across 48 behaviors in nearly 50,000 participants from ten countries (Hall and Rossi, 2008). These studies evidence unusually high precision for behavior change science research (i.e., 1 SD increase in the Pros, and a .5 SD decrease in the Cons across studies). In TTM Interventions, raising one's Pros to change is essential for initiating their movement out of Precontemplation (Prochaska and Prochaska, 2016). The tailoring of Pros and Cons to updated population health information (e.g., smoking puts you at risk for COVID-19) is used to help populations more rapidly detect the benefit of an essential behavior change. As individuals move further through the Stages of Change, decreases in Cons typically follow and by the time they are in Action they typically weight more pros than cons to changing (Prochaska, 1994). The changing pros/cons not only reflect a (self-reported) readiness to engage in intervention events, but also reflect how the individual can detect the costs and benefits of a behavior change. When the interventions assess that the individual is ready to move from one stage to the next, the intervention coordinates new, stage-dependent, behavior change events (e.g., seek social supports in Contemplation, remove cues for smoking during Preparation, begin cessation in Action). TTM interventions support the individual's ability to detect Pros/Cons to Change, while concurrently organizing events that direct the individual through the Stages of Change.

These principles of behavior change support an essential question when conceptualizing behavior change as occurring within a behavioral immune system: What is the relationship between the detection of a problem, and one's direction of change? It seems reasonable to conceive that when the biological immune system does not detect a threatening invader, the probability of developing an infectious disease increases. However, the TTM has helped us to see that this also occurs in health behavior. Prochaska et al. (1994) demonstrated that when a health risk behavior (e.g. smoking addiction) is not weighted as problematic (i.e., weighting more cons than pros to changing chronic smoking), individuals typically remain in Precontemplation. Research clearly demonstrates that individuals that continue health risk behaviors over time (therefore remaining in Precontemplation) dramatically increase their likelihood of developing a chronic disease (Mokdad et al., 2018; Li et al., 2018). Prochaska and Prochaska (2016) emphasize that when intervening on populations in Precontemplation, it is problematic and costly to deliver action-based interventions. If one cannot detect strong enough benefits of change, why try? Thus, the detection of the Pros, is necessary for functional change to be initiated by the individual in the direction of health and wellbeing. Dysfunctional detection (i.e., weighting more Cons than Pros to change a risk behavior) can create and maintain movement in the direction of disease-and-limitation.

Selection in existing behavior change research: the processes of change in the TTM. Human decision-making is constantly impacted by fluctuations in environmental, social, technological, personal and physiological conditions. The TTM assesses and intervenes on Processes of Change that support the intentional Selection of functional conditions that maintain movement through the Stages (despite fluctuations in environmental, social, technological, personal and physiological conditions). Early research on the model sought to support the individual's ability to maintain functional change after an intervention is complete. This meant that its interventions should facilitate change without creating dependency on the intervention to maintain change. To address this challenge during early generations of their model, TTM researchers identified common processes of change in "naive self-changers" (i.e., those who changed without being a part of a structured intervention). Researchers found that selfchangers used common processes that existed in various psychotherapy systems (DiClemente and Prochaska, 1982; DiClemente et al., 1985).

Prochaska et al. (1998) then psychometrically validated ten Processes of Change: Consciousness Raising, Dramatic Relief, Environmental Reevaluation, Social Liberation, Self Reevaluation, Stimulus Control, Helping Relationship, Counter Conditioning, Reinforcement Management, Self-Liberation. Within these ten processes, two second order factors were established. The first five processes (i.e., Consciousness Raising, Dramatic Relief, Environmental Reevaluation, Social Liberation, Self Reevaluation) were termed the Experiential Processes of Change because they were 
evidence of changes in affect and cognition. The second five Processes (Stimulus Control, Helping Relationship, Counter Conditioning, Reinforcement Management, Self-Liberation) were termed the Behavioral Processes of Change because they were evidence of active changes in behavior. The Experiential Processes are emphasized first, because cognitive, affective, and environmental conditions help prepare the reinforcers of functional decision-making. Once the conditions for maintained change are prepared, the self-conditioning, Behavioral Processes are targeted to develop and employ decisions that actively replace bad habits with healthy habits (Prochaska and Prochaska, 2016).

In the same way that enzymes and antibodies tag pathogenic invaders, Experiential Processes "tag" conditions in one's affect, cognition, and environment. For example, Consciousness Raising involves increasing one's awareness of causes, consequences, and outcomes relating to one's behavior. Environmental Reevaluation focuses one's awareness to environmental conditions that influence health risk behaviors. In the same way that antibodies and enzymes also function to actively neutralize/remove pathogens (e.g., dissolving cell walls of bacteria, damaging virus envelopes), the Behavioral Processes function to actively neutralize and remove dysfunctional behavior by selecting for new, functional behavior. For example, Stimulus Control actively removes cues for dysfunctional behavior and adds prompts for functional alternatives. Counterconditioning selects functional behaviors that actively replace dysfunctional behaviors with regularity (e.g., rather than go on a cigarette break after lunch, go on a walk). The TTM research demonstrates that Experiential and Behavioral Processes are both necessary to maintain change longterm (Norman et al., 1998; Redding et al., 2011; Prochaska et al., 1991; Lipschitz et al., 2015; Yusufov et al., 2016).

Consistent decision-making that selects for functional behavior requires preparatory shifts in environmental conditions that reinforce good decisions (e.g., having supporting relationships, being aware of triggers in the environment). However, TTM research demonstrates that experiential conditions for change, are not sufficient for maintained change and the prevention of relapse. Rather, maintained change requires the active selection of healthy habits well. TTM researchers have found that those that initiate change but relapse, typically do not increase reliance on the Behavioral Processes of Change; while, successful changers increase reliance on the Behavioral Processes, particularly emphasizing Counterconditioning and Stimulus Control (Norman et al., 1998; Redding et al., 2011; Prochaska et al., 1991; Lipschitz et al., 2015; Yusufov et al., 2016). This research is vital for population-level behavior change because it demonstrates that experiential/environmental and behavioral conditions both play a role in long-term healthy functioning.

When a bad habit goes away a space remains. When the space remains unfilled, old ways can return. To maintain functional change, one must fill that space by selecting for variations in habit that support maintainable-ease of functioning.

\section{Discussion: the logic of biological and behavioral immunity}

The COVID-19 pandemic has provided clear evidence that the population's immunity during a crisis, its Herd Immunity, is impacted by vulnerabilities of the population to both chronic and acute disease. If one uses the ABIS model, then one can observe a new logic of change that can be used to assess vulnerabilities in population health. This logic-a tri-part logic depicted in Fig. 2can be used to assess the readiness of behavioral and biological immune systems. At the level of one individual person, at the level of one-Self, this tri-part logic can be observed during health behavior change:

1. If one intends to begin a behavior change; then one's Decisions can become ready to Detect the Pros and Cons of a habitual pattern of behavior.

2. If one can detect a point of reference from which to change (e.g. the detected costs of an old habit);

then Decisions can become ready to Direct events that make a change from old habits, toward a new healthy habits.

3. If the new habits can support a healthy lifestyle;

then Decisions can become ready to Select for healthy behaviors that maintain that lifestyle over time.

If this pathway to health is reinforced through detection, direction and selection, then one can become ready to maintain healthy states across time. Notice here in the series of if-then statements, that the functions help to integrate decision-making and habits to form healthy behavior. One's integration of decisions and habits form one's Adaptive Behavioral Immune System. This immune system maintains health at the level of one-Self. At the level of the Cell, this tri-part logic of change can be seen in the biological immune system.

1. If a Biological Immune System is able to maintain immunity, then the Host Defense System can become ready to Detect the costs and benefits of new variations in the microbiome.

i. For example, in a functional immune system, receptors detect if a pathogenic or symbiotic microorganism is present in the body (Hoebe et al., 2004; Zakharova, 2009; Takeuchi and Akira, 2010). Their functioning is instrumental for initiating a response to a pathogen. Given that virus that caused COVID-19 was novel to the human immune system, many immune systems were not ready to efficiently detect the virus's costs to initiate a rapid response.

2. If Host Defense receptors detect a pathogen, then the Host Defense System can become ready to Direct sequences of events that change infectious conditions in the body.

i. For example, COVID-19 vaccines use mRNA to provide directions to the immune system about how to make the spike proteins on the surface of the virus that causes COVID-19 (CDC, 2020). The mVRNA is one source of direction that impacts how the immune system sequences immune events to confer immunity.

3. If this new pathway supports immunity, then the Host Defense System can become ready to Select for conditions of the body that maintain immunity over time (e.g., by releasing antibodies the prevent infection).

i. For example, following exposure to mRNA COVID-19 vaccines, the body releases antibodies that (ideally) will sustain immunity (CDC, 2020).

One question about selection in the biological immune system remains for researchers investigating COVID-19 Vaccines. Will the intervention last? If the intervention lasts, then the system becomes ready to maintain health when the virus is still present. If the intervention does not last, then the immune system requires further intervention to maintain population health. Notice here in the series of if-then statements, that the functions help to integrate host-defense responses with changes to the body that build biological immunity. Researchers can use this tri-part logic to integrate information on the functional or dysfunctional states of immune systems.

Future directions of the ABIS: messaging in the population. In addition to aiding integration of research, parallels among 

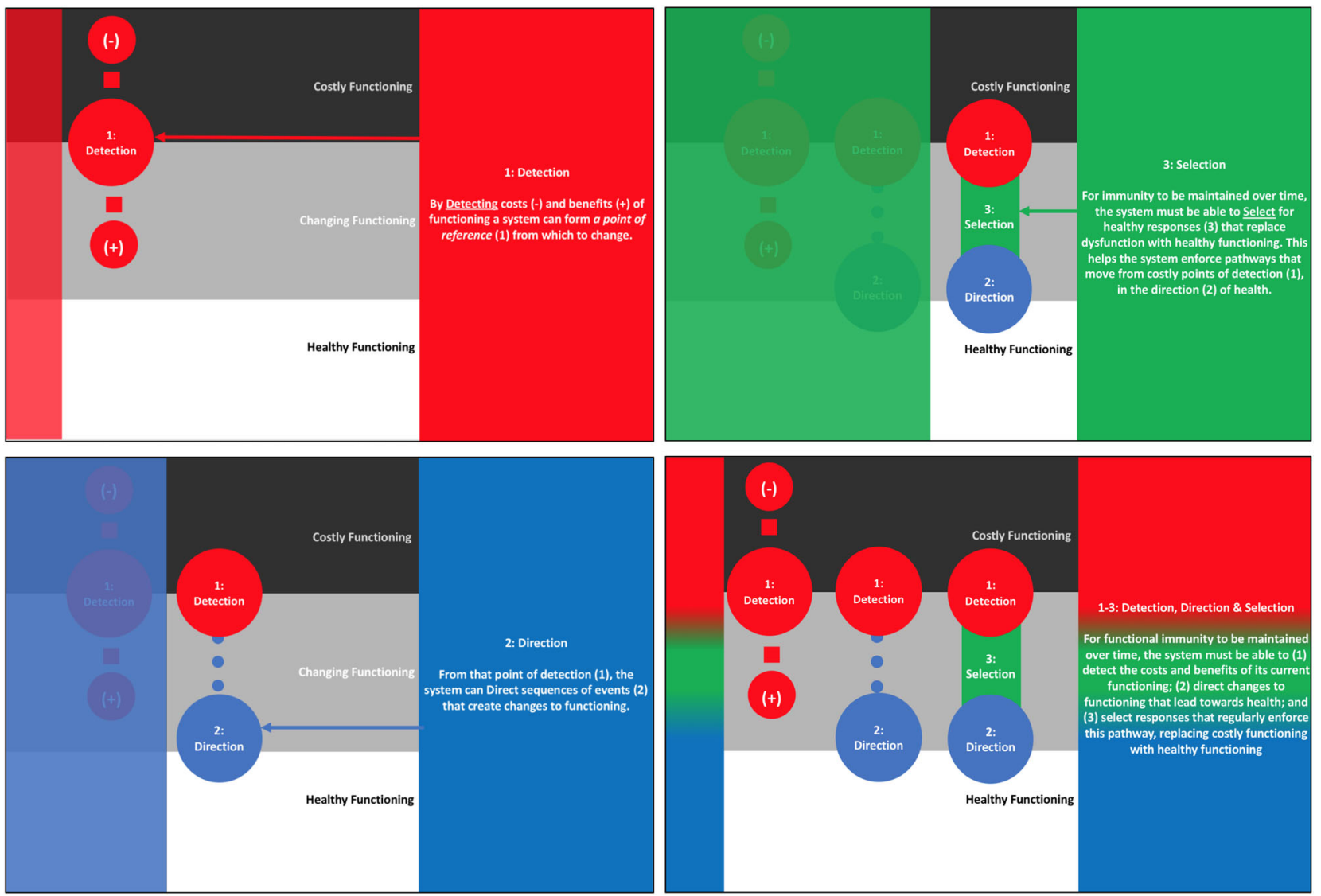

Fig. 2 The path of precision. This model of health demonstrates how precision functions integrate to organize immunity. Functional precision organizes variation. Functional variation can be maintained when functional precision detects, directs and selects for healthy change. When integrated, the precision functions serve health from multiple levels.

biological and behavioral immune systems can be helpful when creating messaging about population health. The tangible parallels among biological and behavioral immune systems can be helpful when tailoring intervention-messages to populations. For example, not all in the population can immediately understand the function of immune receptors. However, more in the population will be ready to understand how interventions support their ability detect the pros and cons, the costs and benefits, of changes in health. When creating messages about the important steps towards population immunity, researchers can use the logic to explain interventions:

Well-dosed vaccines (e.g., the COVID-19 Vaccine) support the biological immune system's ability to:

1. detect a new virus;

2. organize a helpful sequence of biological responses to the virus; and.

3. release antibodies to support those responses over time.

Well-tailored behavior change interventions (e.g., by using the Stages of Change and Decisional Balance from the TTM) support one's ability to:

1. detect the Pros and Cons of a behavior,

2. organize a helpful sequence changes that lead to a healthy lifestyle, and.

3. select for conditions that maintain that healthy lifestyle.

By communicating information within a single framework, a reasoning of prevention and intervention can become more apparent to the general public.
Future directions of the ABIS: new questions in population health. The ABIS model can be used to develop research questions about population health. For example, "How do interventions upon behavioral immunity also support biological immunity during acute COVID-19 infection?" During the initial stages of the COVID-19 pandemic, doctors discovered that turning patients on their belly allowed them to maintain their breathing when severely suffering from COVID-19. Prior to this discovery doctors were primarily using ventilators as part of an intervention on breathing. While ventilators might be limited on hospital floors during a crisis, a person's body weight is readily available. By intervening on breathing (i.e., intervening on behavioral immunity), many could stay alive to fight the COVID19 infection (i.e., supporting biological immunity).

In addition to keeping a person alive, research also suggests that breathing patterns also modulate immune cell activity (Asimakos et al., 2018). Therefore, the proper timing of precise interventions on behavioral immunity can also impact biological immunity. Further, the proper timing of behavioral prevention methods can support biological immunity in the long run. Exercise practices that stimulate vigorous breathing are protective of the biological immune system (Jesus et al., 2021). If healthy breathing methods (as well as moving, eating and drinking methods) support immunity, then how might nationwide prevention initiatives support population health? Public initiatives might consider new, researchable questions:

- "What is the relationship between host-defense activity (i.e., precision of biological immunity) and habitual variations of breathing (i.e., variation of behavioral immunity)? 
- What is the relationship between host-defense activity (i.e., precision of biological immunity) and habitual variations of drinking, eating and moving (i.e., variation of behavioral immunity)?"

- What is the relationship between host-defense activity (i.e., precision of biological immunity), habitual patterns of breathing and moving (i.e., variation of behavioral immunity) and mindfulness practice (i.e., precision of behavioral immunity) seen during yoga?"

While these research questions are not systematically investigated in Western Medical Science, they are central to the execution of Eastern Medical Science. Behavioral changes are typically prescribed following the diagnosis of a condition. Eastern methods of breathing and moving (e.g., pranayama yoga in Ayurvedic Medicine, qigong in Traditional Chinese Medicine) and stress-management (e.g., Mindfulness Meditation) are a part of health interventions. As healthcare facilities continue to integrate behavioral health into medical practice, there remains the need for an accepted model that will integrate these healthcare practices. The ABIS model is one model that can get the job done.

Future directions of the ABIS: an integrated language of health. The functions in the ABIS model create a language of health that can help researchers and providers integrate biological and behavioral processes. In this paper, sustainable habits and sustainable changes to the human microbiome are reflected in states of variability. Variability in habits (i.e., healthy/wellbalanced life style) and in the microbiome are sustained through the precision of decision-making and the host-defense system, respectively. By viewing the forms of precision (e.g., decisions and host-defense) as they detect, direct, and select for functional variability; we can more logically organize discussions of how long-term change emerges within Cells and Selves. For example:

1. If cells and selves become increasingly able to vary (i.e., increased variation); and become increasingly able to organize in varying conditions (i.e., increased precision); then immunity emerges.

2. If cells and selves become increasingly able to organize following exposure to varying conditions; then the system's variability becomes a reflection of the adaptations to the changing environment.

3. If adaptation to the changing environment is maintained, then the system's capacity for organization (i.e., its precision) is reinforced by the changing environment.

While populations cannot control what they are exposed to in the environment (e.g., as seen during the COVID-19 pandemic); they can facilitate methods that strengthen the immune functions cited. A goal of this paper is to provide a logic (depicted in Fig. 2) of how immunity builds.

When the population builds immunity in rapidly changing environments, the individuals in the population retain more of their freedom to make personal decisions. When population immunity suffers, certain personal freedoms become sacrificed so that the health of the whole is not sacrificed. When the individual is better able to make healthy decisions during a stressful time, their biological immune system can be supported by healthy behavior. When the host-defense system is able to prevent the spread of infectious disease, human beings become freer to make decisions. The COVID-19 pandemic has been strong evidence of a clash between the wester ideal of (Personal) Freedom and the universal value of (Population) Health. The ABIS model provides a way of viewing the impact of these Values from multiple levels. How will we move forward and value Population Health and Personal Freedom in the twenty-first century? That is a question for a future paper. Perhaps it is a question for "A Social Immune System".

\section{Data availability}

Data sharing not applicable to this article as no datasets were generated or analyzed during the current study.

Received: 6 May 2020; Accepted: 23 February 2021;

Published online: 29 March 2021

\section{References}

Ader R (1980) Presidential address: Psychosomatic and psychoimmunological research. Psychosom Med 42:307-21

Ader R, Cohen N (1974) Behaviorally conditioned immunosuppression. Psychosom Med 37(4):333-340

Armitage CJ, Conner M (2000) Social cognition models and health behaviour: structured review. Psychol Health 15(2):173-189

Asimakos A, Toumpanakis D, Karatza MH, Vasileiou S, Katsaounou P, Mastora Z, Vassilakopoulos $T$ (2018) Immune cell response to strenuous resistive breathing: comparison with whole body exercise and the effects of antioxidants. Int J Chron Obstruct Pulmon Dis 13:529

Bandura A (1965) Influence of models' reinforcement contingencies on the acquisition of imitative responses. J Pers Soc Psychol 1(6):589

Berrigan D, Dodd K, Troiano RP, Krebs-Smith SM, Barbash RB (2003) Patterns of health behavior in US adults. Prevent Med 36(5):615-623

Blaut M, Clavel T (2007) Metabolic diversity of the intestinal microbiota: implications for health and disease. J Nutr 137(3):751S-755S

Bonneaud C, Mazuc J, Gonzalez G, Haussy C, Chastel O, Faivre B, Sorci G (2003) Assessing the cost of mounting an immune response. Am Nat 161 (3):367-379

CDC (2018) History of smallpox. Centers for Disease Control and Prevention. Aug Retrieved from https://www.cdc.gov/smallpox/history/history.html

CDC (2020) Understanding mRNA COVID-19 vaccines. Centers for Disease Control and Prevention. Dec Retrieved from https://www.cdc.gov/coronavirus/2019ncov/vaccines/different-vaccines/mrna.html

Chartier G, Cawthorpe D (2016) From 'Big 4'to 'Big 5': a review and epidemiological study on the relationship between psychiatric disorders and World Health Organization preventable diseases. Curr Opin Psychiatr 29 (5):316-321

Cheng VC, Wong SC, Chuang VW, So SY, Chen JH, Sridhar S, To KK, Chan JF, Hung IF, Ho PL, Yuen KY (2020) The role of community-wide wearing of face mask for control of coronavirus disease 2019 (COVID-19) epidemic due to SARS-CoV-2. J Infection 81(1):107-114

Clark JA, Fessler DM (2014) Recontextualizing the behavioral immune system within psychoneuroimmunology. Evol Behav Sci 8(4):235

Clark DA, Beck AT (2011) Cognitive therapy of anxiety disorders: Science and practice. Guilford Press

Clemente JC, Ursell LK, Parfrey LW, Knight R (2012) The impact of the gut microbiota on human health: an integrative view. Cell 148(6):1258-1270

Conner M, Sparks P (2005) Theory of planned behaviour and health behaviour. In: Conner M, Norman P (eds) Predicting health behaviour, 2nd edn. Open University Press. pp. 170-222

D’Angelo H, Ammerman A, Gordon-Larsen P, Linnan L, Lytle L, Ribisl KM (2016) Sociodemographic disparities in proximity of schools to tobacco outlets and fast-food restaurants. Am J Public Health 106(9 Sep):1556-62. https://doi. org/10.2105/AJPH.2016.303259. Epub 26 July 2016

Dantzer R (2009) Cytokine, sickness behavior, and depression. Immunol Allergy Clin North Am 29(2):247-264. https://doi.org/10.1016/j.iac.2009.02.002

Davison GC (1968) Systematic desensitization as a counterconditioning process. J Abnorm Psychol 73(2):91

Dethlefsen L, McFall-Ngai M, Relman DA (2007) An ecological and evolutionary perspective on human-microbe mutualism and disease. Nature 449(7164):811

De Vadder F, Kovatcheva-Datchary P, Goncalves D, Vinera J, Zitoun C, Duchampt A, Mithieux G (2014) Microbiota-generated metabolites promote metabolic benefits via gut-brain neural circuits. Cell 156(1-2):84-96

DiClemente CC, Prochaska JO (1982) Self-change and therapy change of smoking behavior: a comparison of processes of change in cessation and maintenance. Addict Behav 7(2):133-142

DiClemente CC, Prochaska JO, Gibertini M (1985) Self-efficacy and the stages of self-change of smoking. Cognit Ther Res 9(2):181-200

Drela N, Kozdron E, Szczypiorski P (2004) Moderate exercise may attenuate some aspects of immunosenescence. BMC Geriatr 4(1):1-7

Dreyzehner J (2017) The Big 4: using primary prevention to drive population health. J Public Health Manage Pract 23(1):1

Eikenberry SE, Mancuso M, Iboi E, Phan T, Eikenberry K, Kuang Y, Kostelich E, Gumel AB (2020) To mask or not to mask: Modeling the potential for face 
mask use by the general public to curtail the COVID-19 pandemic. Infect Dis Model 5:293-308

Hall KL, Rossi JS (2008) Meta-analytic examination of the strong and weak principles across 48 health behaviors. Prevent Med 46(3):266-274

Hall SM, Tunstall CD, Vila KL, Duffy J (1992) Weight gain prevention and smoking cessation: cautionary findings. Am J Public Health 82(6):799-803

Hoebe K, Janssen E, Beutler B (2004) The interface between innate and adaptive immunity. Nat Immunol 5(10):971

Janeway Jr. CA (1992) The immune system evolved to discriminate infectious nonself from noninfectious self. Immunol Today 13(1):11-16

Janeway Jr CA, Medzhitov R (2002) Innate immune recognition. Ann Rev Immunol 20(1):197-216

Janeway Jr CA, Travers P, Walport M, Shlomchik MJ (2014) Immunobiology: the immune system in health and disease. Garland Science, New York, NY, pp. S620-626

Janis IL (1959) Decisional conflicts: a theoretical analysis. J Conflict Resolut 3 (1):6-27

Janis IL, Mann L (1976) Coping with decisional conflict: An analysis of how stress affects decision-making suggests interventions to improve the process. Am Sci 64(6):657-667

Janz NK, Becker MH (1984) The health belief model: a decade later. Health Educ Q 11(1):1-47

Jesus I, Vanhee V, Deramaudt TB, Bonay M (2021) Promising effects of exercise on the cardiovascular, metabolic and immune system during COVID-19 period. J Hum Hypertens 35(1):1-3

Katz DL (2009) School-based interventions for health promotion and weight control: not just waiting on the world to change. Ann Rev Public Health 30:253-272

Katz DL, Frates EP, Bonnet JP, Gupta SK, Vartiainen E, Carmona RH (2017) Lifestyle as medicine: the case for a true health initiative. Am J Health Promot 32(6):1452-1458

Katz DL, O'Connell M, Yeh MC, Nawaz H, Njike V, Anderson LM, Dietz W (2005) Public health strategies for preventing and controlling overweight and obesity in school and worksite settings: a report on recommendations of the Task Force on Community Preventive Services. MMWR Recomm Rep 54(10):1-12

Kent S, Bluthé RM, Kelley KW, Dantzer R (1992) Sickness behavior as a new target for drug development. Trend Pharmacol Sci 13:24-28

Kretzer EK, Larson EL (1998) Behavioural interventions to improve infection control practices. Am J Infect Control 26(3):245-253

Li Y, Pan A, Wang DD, Liu X, Dhana K, Franco OH, Hu FB (2018) Impact of healthy lifestyle factors on life expectancies in the US population. Circulation 137:345-355

Linehan M (2014). DBT? Skills training manual. Guilford Publications

Lippke S, Nigg CR, Maddock JE (2012) Health-promoting and health-risk behaviors: theory-driven analyses of multiple health behavior change in three international samples. Int J Behav Med 19(1):1-13

Lipschitz JM, Yusufov M, Paiva A, Redding CA, Rossi JS, Johnson S, Prochaska JO (2015) Transtheoretical principles and processes for adopting physical activity: a longitudinal 24-month comparison of maintainers, relapsers, and nonchangers. J Sport Exerc Psychol 37(6):592-606

Mantzari E, Vogt F, Shemilt I, Wei Y, Higgins JP, Marteau TM (2015) Personal financial incentives for changing habitual health-related behaviors: a systematic review and meta-analysis. Prevent Med 75:75-85

Medzhitov R, Janeway CA (2002) Decoding the patterns of self and nonself by the innate immune system. Science 296(5566):298-300

Mokdad AH (2004) Actual causes of death in the United States, 2000. JAMA 291(10):1238

Mokdad AH, Ballestros K, Echko M, Glenn S, Olsen HE, Mullany E, Kasaeian A (2018) The state of US health, 1990-2016: burden of diseases, injuries, and risk factors among US states. JAMA 319(14):1444-1472

Moulin AM (1989) The immune system: a key concept for the history of immunology. Hist Philos Life Sci 11:221-236

Naor D, Tarcic N (1982) Control of autoimmune responses induced with modified self antigens. Ann New York Acad Sci 392:178-190

Norman GJ, Velicer WF, Fava JL, Prochaska JO (1998) Dynamic typology clustering within the stages of change for smoking cessation. Addict Behav 23(2):139-153

Ornish D, Scherwitz LW, Billings JH, Gould KL, Merritt TA, Sparler S, Brand RJ (1998) Intensive lifestyle changes for reversal of coronary heart disease. JAMA 280(23):2001-2007

Pérez-Brocal V, Latorre A, Moya A (2011) Symbionts and pathogens: What is the difference? Between Pathogenicity and Commensalism, pp. 215-243

Prochaska JJ, Prochaska JO (2011) A review of multiple health behavior change interventions for primary prevention. Am J Lifestyle Med 5(3):208-221

Prochaska JO (1994) Strong and weak principles for progressing from precontemplation to action on the basis of twelve problem behaviors. Health Psychol 13(1):47

Prochaska JO, DiClemente CC (1983) Stages and processes of self-change of smoking: toward an integrative model of change. J Consult Clin Psychol 51(3):390-95
Prochaska JO, Norcross JC, Saul SF (2020) Generating psychotherapy breakthroughs: Transtheoretical strategies from population health psychology. American Psychologist 75(7):996-1010

Prochaska JO, Velicer WF, DiClemente CC, Fava J (1988) Measuring processes of change: Applications to the cessation of smoking. J Consult Clin Psychol 56(4):520-528

Prochaska JO, Velicer WF, Guadagnoli E, Rossi JS, DiClemente CC (1991) Patterns of change: dynamic typology applied to smoking cessation. Multivariate Behav Res 26(1):83-107

Prochaska JO, Velicer WF, Rossi JS, Goldstein MG, Marcus BH, Rakowski W, Rossi SR (1994) Stages of change and decisional balance for 12 problem behaviors. Health Psychol 13(1):39

Prochaska JO, Prochaska JM (2016) Changing to thrive: using the stages of change to overcome the top threats to your health and happiness. Simon and Schuster

Råberg L, Nilsson JÅ, Ilmonen P, Stjernman M, Hasselquist D (2000) The cost of an immune response: vaccination reduces parental effort. Ecol Lett 3 (5):382-386

Redding CA, Prochaska JO, Paiva A, Rossi JS, Velicer W, Blissmer BJ, Sun X (2011) Baseline stage, severity, and effort effects differentiate stable smokers from maintainers and relapsers. Subst Use Misuse 46(13):1664-1674

Rosenstock IM (1974) Historical origins of the health belief model. Health Educ Monogr 2(4):328-335

Rothe M, Blaut M (2012) Evolution of the gut microbiota and the influence of diet. Benef Microbes 4(1):31-37

Ruíz-Argüelles A (1996) The cascade of the immune response. Curr Ther Res 57 (13):8-13

Saad JM, Prochaska JO (2020) A philosophy of health: life as reality, health as a universal value. Palgrave Commun 6(1):1-1

Schaller M, Duncan LA (2007) The behavioral immune system: its evolution and social psychological implications. In: Forgas JP, Haselton MG, von Hippel W (eds.) Sydney symposium of social psychology. Evolution and the social mind: Evolutionary psychology and social cognition. Routledge/Taylor \& Francis Group, New York, NY, US, pp. 293-307

Schaller M, Park JH (2011) The behavioral immune system (and why it matters). Curr Dir Psychol Sci 20(2):99-103

Schaller M, Duncan LA (2016) The behavioral immune system. The handbook of evolutionary psychology 1:206-224

Schwarzer R (2008) Modeling health behavior change: how to predict and modify the adoption and maintenance of health behaviors. Appl Psychol 57(1):1-29

Skinner BF (1953) Science and human behavior. Macmillan, New York, NY

Solomon GF (1969) Emotions, stress, the central nervous system, and immunity. Ann N Y Acad Sci 164(2):335-343

Solomon GF (1981) Psychoneuroendocrinological effects on the immune response. Ann Rev Microbiol 35(1):155-184

Solomon GF, Moos RH (1964) Emotions, immunity, and disease: a speculative theoretical integration. Arch Gen Psychiat 11(6):657-674

Solomon GF, Moos RH (1965) The relationship of personality to the presence of rheumatoid factor in asymptomatic relatives of patients with rheumatoid arthritis. Psychosom Med 27(4):350-360

Steinberg CM, Di Lorenzo C Lefkovits I (eds.) (1981) The Immune system: festschrift in honor of Niels Kaj Jerne on the occasion of his 70th birthday: past and future. Karger

Sutton S (1998) Predicting and explaining intentions and behavior: how well are we doing? J Appl Soc Psychol 28(15):1317-1338

Sutton S (1994) The past predicts the future: interpreting behaviour-behaviour relationships in social psychological models of health behaviour. APA

Takeuchi O, Akira S (2010) Pattern recognition receptors and inflammation. Cell 140(6):805-820

Thaler RH (2018) From cashews to nudges: the evolution of behavioral economics. Am Econ Rev 108(6 Jun):1265-87. 2018

Velicer WF, DiClemente CC, Prochaska JO, Brandenburg N (1985) Decisional balance measure for assessing and predicting smoking status. J Pers Soc Psychol 48(5):1279

Wolpe J (1961) The systematic desensitization treatment of neuroses. J Nerv Ment Dis 132(3):189-203

Wolpe J (1968) Psychotherapy by reciprocal inhibition. Cond Reflex 3(4):234-240 Xia C, Wang L, Sun S, Wang J (2012) An SIR model with infection delay and propagation vector in complex networks. Nonlinear Dyn 69(3):927-934

Yusufov M, Paiva AL, Redding CA, Lipschitz JM, Gokbayrak NS, Greene G, Prochaska JO (2016) Fat reduction efforts: a 24-month longitudinal comparison of a large sample of maintainers, relapsers, and non-changers. Health Promot Pract 17(1):116-126

Zakharova LA (2009) Evolution of adaptive immunity. Biol Bull 36(2):107-116

\section{Acknowledgements}

This research was not supported by funding. 


\section{Competing interests}

The authors declared the following potential competing interests: JOP, Ph.D. is a founder of the Transtheoretical Model (TTM) of Behavior Change, and is the Director of the Cancer Prevention Research Center at the University of Rhode Island. JMS, M.Ed. is currently a Ph.D. student conducting dissertation research on population health and behavior change under Dr. Prochaska's supervision at the University of Rhode Island

\section{Additional information}

Correspondence and requests for materials should be addressed to J.M.S.

Reprints and permission information is available at http://www.nature.com/reprints

Publisher's note Springer Nature remains neutral with regard to jurisdictional claims in published maps and institutional affiliations. (c) (i) Open Access This article is licensed under a Creative Commons Attribution 4.0 International License, which permits use, sharing, adaptation, distribution and reproduction in any medium or format, as long as you give appropriate credit to the original author(s) and the source, provide a link to the Creative Commons license, and indicate if changes were made. The images or other third party material in this article are included in the article's Creative Commons license, unless indicated otherwise in a credit line to the material. If material is not included in the article's Creative Commons license and your intended use is not permitted by statutory regulation or exceeds the permitted use, you will need to obtain permission directly from the copyright holder. To view a copy of this license, visit http://creativecommons.org/ licenses/by/4.0/.

(C) The Author(s) 2021 